EPJ Web of Conferences 36, 00020 (2012)

DOI: $10.1051 /$ epjconf/20123600020

(C) Owned by the authors, published by EDP Sciences, 2012

\title{
Hadronic Resonances from STAR
}

\author{
Masayuki Wada (for the STAR collaboration) \\ ${ }^{1}$ The University of Texas at Austin
}

\begin{abstract}
The results of resonance particle productions $\left(\rho^{0}, \omega, K^{*}, \phi, \Sigma^{*}\right.$, and $\left.\Lambda^{*}\right)$ measured by the STAR collaboration at RHIC from various colliding systems and energies are presented. Measured mass, width, $\left\langle p_{T}\right\rangle$, and yield of those resonances are reviewed. No significant mass shifts or width broadening beyond the experiment uncertainties are observed. New measurements of $\phi$ and $\omega$ from leptonic decay channels are presented. The yields from leptonic decay channels are compared with the measurements from hadronic decay channels and the two results are consistent with each other.
\end{abstract}

\section{Introduction}

Ultra-relativistic heavy ion collisions are an effective way to create hot and dense strongly interacting matter in laboratories. Hadronic resonance particles can be used as probes to study this strongly interacting matter because of their short lifetimes compared to that of the fireball. In-medium modification of their masses and widths are expected in hot and dense mediums [1,2]. If those modifications exist, it could possibly indicate chiral symmetry restoration. In hadronic decay channels, yield and momentum spectra of resonances may be modified due to resonance decay and the subsequent re-scattering of their decay daughters as well as the re-generation of resonances during the fireball lifetime. By contrast, di-leptons can probe the whole evolution of the collision without the hadronic medium effects at the later stage because of the relatively small cross section of leptons with the hadronic medium. Combined with ionization energy loss (dE/dx) information from the Time Projection Chamber (TPC), the newly installed Time of Flight detector (TOF) [3] provides a clean particle identification (PID) at low and intermediate momentum $(\mathrm{p}=0.2-2.0 \mathrm{GeV} / \mathrm{c})$, which enable us to measure resonance particles through the rare di-electron channel. A difference in resonance yields and momentum spectra in heavy ion collisions from hadronic and leptonic decay channels may show the signatures of the initial production of resonances which are probably washed out in hadronic decay channels but remains in leptonic decay channels. The $\phi$ meson is one of the resonance particles of which the measurements from both leptonic and hadronic decay channels are possible.

Data shown here were measured by the STAR collaboration at RHIC. In STAR, many resonance particles are measured in several collision systems $(p+p, d+A u, C u+C u$, and $A u+A u)$ and energies (only $\sqrt{s_{N N}}=62.4$ and $200 \mathrm{GeV}$ are presented). In this paper, we summarize those results by comparing the masses, widths, $\mathrm{dN} / \mathrm{dy}$, and mean transverse momentum of resonance particles from different collision systems and energies. In Table 1, the Particle Data Group (PDG) values [4] for the mass, width, lifetime, and decay channels of hadronic resonances are summarized.

\section{Resonances from hadronic decay channels}

\subsection{Masses and Widths}

In this section, we focus on the differences of the masses and widths among collision systems and energies.

This is an Open Access article distributed under the terms of the Creative Commons Attribution License 2.0, which permits unrestricted use, distribution, and reproduction in any medium, provided the original work is properly cited. 
Table 1: Resonances from PDG [4].

\begin{tabular}{lllll}
\hline particle & Mass $\left(\mathrm{MeV} / \mathrm{c}^{2}\right)$ & Width $\left(\mathrm{MeV} / \mathrm{c}^{2}\right)$ & Lifetime $(\mathrm{fm} / \mathrm{c})$ & Decay channel \\
\hline$\rho^{0}(770)$ & $775.49 \pm 0.34$ & $149.1 \pm 0.8$ & 1.32 & $\pi+\pi$ \\
$\omega(782)$ & $782.65 \pm 0.12$ & $8.49 \pm 0.08$ & 23.2 & $e^{+}+e^{-}$ \\
$K^{* 0}(892)$ & $895.94 \pm 0.22$ & $48.7 \pm 0.8$ & 4.05 & $\mathrm{~K}+\pi$ \\
$\phi(1020)$ & $1019.455 \pm 0.020$ & $4.26 \pm 0.04$ & 46.3 & $\mathrm{~K}+\mathrm{K}, e^{+}+e^{-}$ \\
$\Sigma^{*+}(1385)$ & $1382.80 \pm 0.35$ & $36.0 \pm 0.7$ & 5.48 & $\Lambda(\rightarrow p+\pi)+\pi^{+}$ \\
$\Sigma^{*-}(1385)$ & $1387.2 \pm 0.5$ & $39.4 \pm 2.1$ & 5.01 & $\Lambda(\rightarrow p+\pi)+\pi^{-}$ \\
$\Lambda^{*}(1520)$ & $1519.5 \pm 1.0$ & $15.6 \pm 1.0$ & 12.6 & $\mathrm{~K}+\mathrm{p}$ \\
\hline
\end{tabular}

In Fig. 1a, the invariant mass of $\pi^{+} \pi^{-}$after like-sign background subtraction in minimum bias (MB) $\mathrm{p}+\mathrm{p}$ (upper) and peripheral (40-80\% central) $\mathrm{Au}+\mathrm{Au}$ (lower) collisions are presented. The $\rho^{0}$ signal is fitted with a relativistic Breit-Wigner function multiplied by a phase factor with a fixed width of $\Gamma=$ $160 \mathrm{MeV} / \mathrm{c}^{2}$ which is consistent with the $\rho^{0}$ natural width folded with intrinsic detector resolution. The $\rho^{0}$ masses shown in Fig. $1 \mathrm{~b}$ are obtained by the fit as a function of $p_{T}$. The measured $\rho^{0}$ peak in the invariant mass distribution is lower than the PDG value by $\sim 30-70 \mathrm{MeV} / \mathrm{c}^{2}$ in all systems at low $p_{T}$. Effects such as phase space and dynamical interactions with matter may explain this modification of the $\rho^{0}$ mass [7].

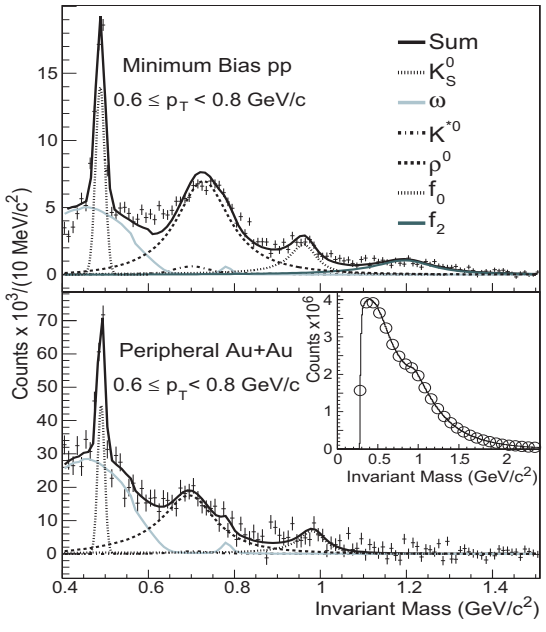

(a) $\pi \pi$ invariant mass distributions [5]

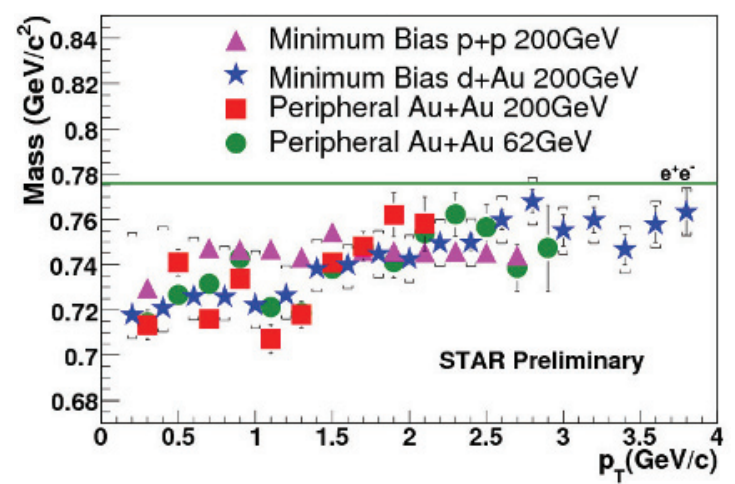

(b) Fit mass vs. $p_{T}[6]$

Fig. 1: $\rho^{0} \rightarrow \pi^{+} \pi^{-}$invariant mass distributions and mass shifts

(a): The invariant mass of $\pi^{+} \pi^{-}$in $\mathrm{p}+\mathrm{p}$ (upper panel) and Au $+\mathrm{Au}$ (lower panel) collisions. The $\rho^{0}$ signals are fit by the relativistic Breit-Wigner function times phase factor with fixed width. (b): The $\rho^{0}$ mass vs. $p_{T}$ in $\mathrm{MB} \mathrm{p}+\mathrm{p}$ and $\mathrm{d}+\mathrm{Au}$ and peripheral $\mathrm{Au}+\mathrm{Au}$ collisions at $\sqrt{s_{N N}}=62.4$ and $200 \mathrm{GeV}$. The green line shows the PDG value of $\rho^{0}$ mass.

In Fig. 2a, the $K \pi$ invariant mass distributions after the mix-event background subtraction in $\mathrm{MB}$ $\mathrm{p}+\mathrm{p}$ and $0-10 \%$ central $\mathrm{Au}+\mathrm{Au}$ collisions at $\sqrt{s_{N N}}=200 \mathrm{GeV}$ are presented. The $K^{* 0}$ signals are fit with a relativistic Breit-Wigner function multiplied by a phase factor. The fit results of the masses and widths as a function of $p_{T}$ are shown in Fig. $2 \mathrm{~b}$ with the Monte Carlo (MC) simulation results which are obtained from the embedded MC tracks by fitting the invariant mass distribution after reconstruction. This means the results include the resolution of the momentum measurement in STAR. The measured 


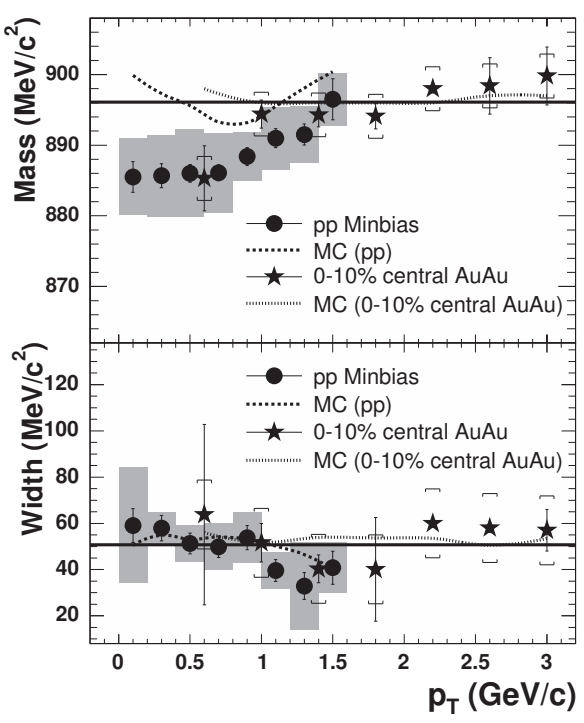

(a) $K \pi$ invariant mass distribution [8]

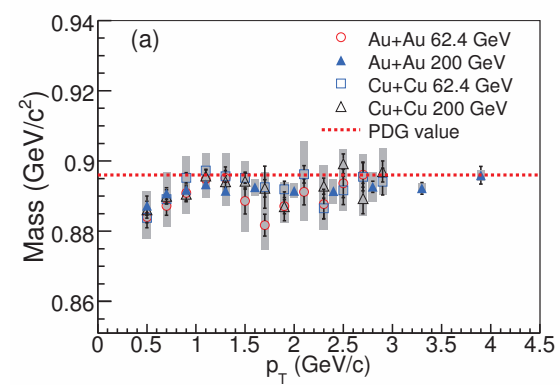

(c) Fit mass [9]

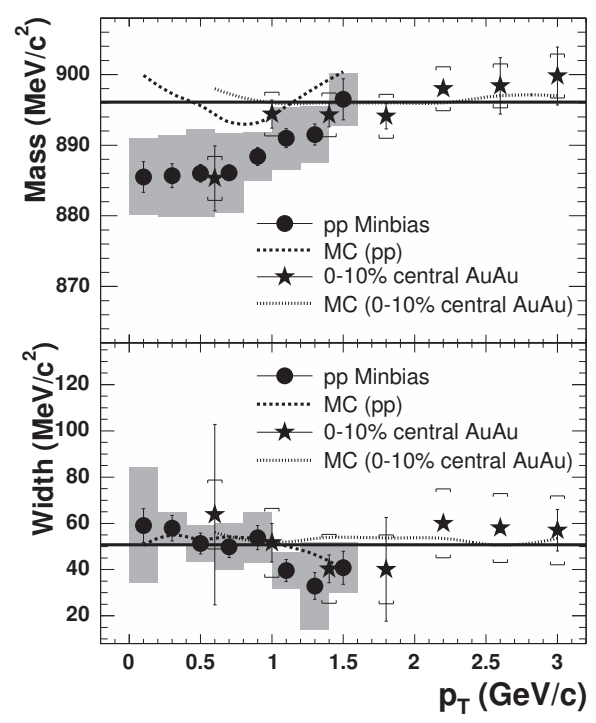

(b) $K^{* 0}$ mass and width [8]

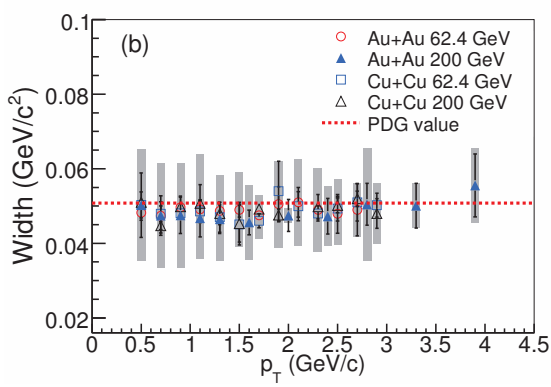

(d) Fit width [9]

Fig. 2: $\mathbf{K}^{* \mathbf{0}} \rightarrow \pi \mathrm{K}$ in $\mathbf{p}+\mathbf{p}, \mathrm{Cu}+\mathrm{Cu}$, and $\mathrm{Au}+\mathrm{Au}$ at $\sqrt{s_{N N}}=\mathbf{6 2 . 4}$ and $200 \mathrm{GeV}$

(a): The invariant mass of $K \pi$ in $\mathrm{p}+\mathrm{p}$ (lower panel) and $\mathrm{Au}+\mathrm{Au}$ (upper panel) collisions at $\sqrt{s_{N N}}=$ $200 \mathrm{GeV}$. (b): The $K^{* 0}$ mass and width in $\mathrm{MB} \mathrm{p}+\mathrm{p}$ and $0-10 \%$ central Au $+\mathrm{Au}$ collisions at $\sqrt{s_{N N}}=$ $200 \mathrm{GeV}$. The solid straight lines are the PDG $K^{* 0}$ values. The dashed and dotted curves are the Monte Carlo simulation results. The grey shadows (caps) indicate the systematic uncertainties for the measurement in $\mathrm{MB} \mathrm{p}+\mathrm{p}$ interactions (central $\mathrm{Au}+\mathrm{Au}$ collisions). (c,d): The $K^{* 0}$ mass and width in $\mathrm{MB} \mathrm{Cu}+\mathrm{Cu}$ and $\mathrm{Au}+\mathrm{Au}$ collisions at $\sqrt{s_{N N}}=62.4$ and $200 \mathrm{GeV}$. The dashed lines represent the PDG values. The error bars and the gray shadows show statistical and systematical uncertainties, respectively.

widths are consistent with the MC and the PDG value in both collision systems. On the other hand, the measured masses show downward shifts at low $p_{T}$ in both $\mathrm{MB} \mathrm{p}+\mathrm{p}$ and $0-10 \%$ central $\mathrm{Au}+\mathrm{Au}$ collisions at a level of $2 \sigma$. Figure 2 lower panel shows the measured masses (2c) and widths (2d) for various $p_{T}$ bins in $\mathrm{Cu}+\mathrm{Cu}$ and $\mathrm{Au}+\mathrm{Au}$ collisions at $\sqrt{s_{N N}}=62.4$ and $200 \mathrm{GeV}$ with the red dashed lines representing PDG values. These values are obtained from fits using the non-relativistic BreitWigner function plus a linear residual background. The measured widths are consistent with the PDG value while the measured masses are within $2 \sigma$ of the PDG value at low $p_{T}$. There is no significant dependence of the $K^{* 0}$ mass and width on the beam energies or the colliding ion species studied. It is unlikely that these shifts are caused by the medium effect. Although pion and kaon tracks are corrected 
for energy loss due to multiple scattering in the TPC, other detector effects may not be fully accounted for.

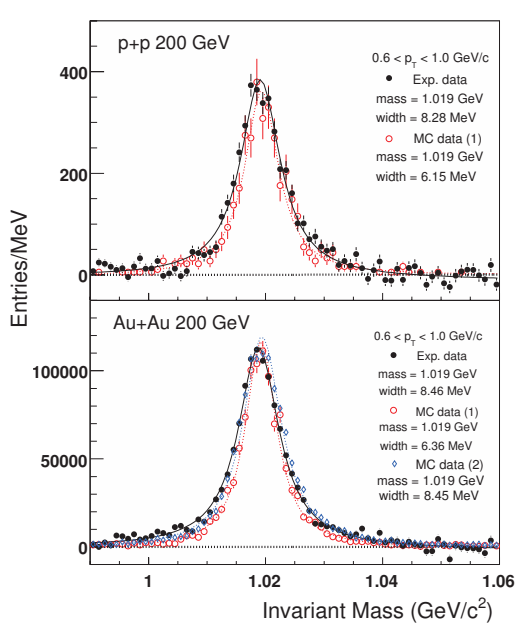

(a) $K^{+} K^{-}$invariant mass distribution [10]

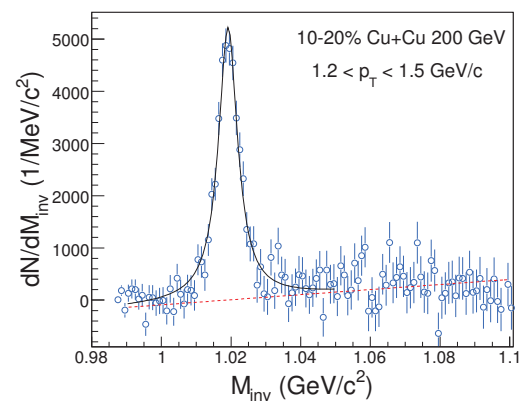

(c) $K^{+} K^{-}$invariant mass distribution in $\mathrm{Cu}+\mathrm{Cu}$ collisions [11]

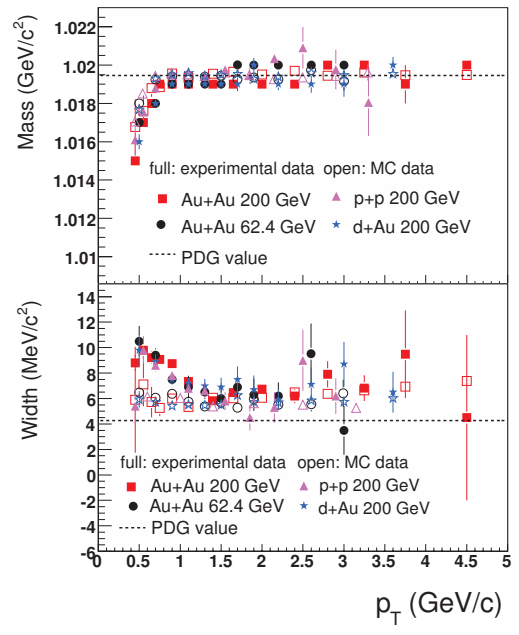

(b) $\phi$ meson mass and width [10]

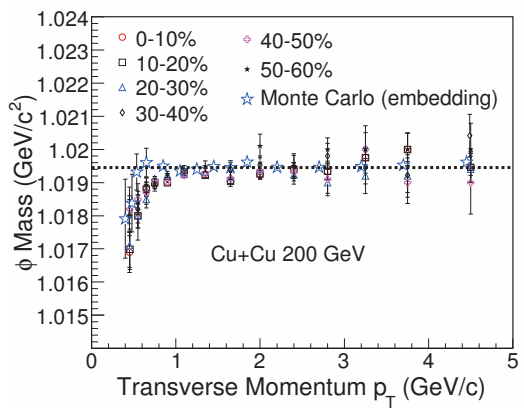

(d) $\phi$ meson width in $\mathrm{Cu}+\mathrm{Cu}$ collisions [11]

Fig. 3: $\phi \rightarrow \mathrm{K}^{+} \mathrm{K}^{-}$in $\mathbf{p}+\mathbf{p}, \mathrm{Cu}+\mathrm{Cu}$, and $\mathrm{Au}+\mathrm{Au}$ at $\sqrt{s_{N N}}=62.4$ and $200 \mathrm{GeV}$

(a): The invariant mass distributions of $K^{+} K^{-}$in $\mathrm{p}+\mathrm{p}$ (upper panel) and $\mathrm{Au}+\mathrm{Au}$ (lower panel) collisions at $\sqrt{s_{N N}}=200 \mathrm{GeV}$. The $\phi$ meson signals are fit with a non-relativistic Breit-Wigner function superimposed on a linear background function. Open and solid symbols respectively represent the experimental data and the MC simulation results. (b): The masses and widths of $\phi$ meson as a function of $p_{T}$ in p + p $200 \mathrm{GeV}$ (NSD), d + Au $200 \mathrm{GeV}(0-20 \%), \mathrm{Au}+\mathrm{Au} 62.4 \mathrm{GeV}(0-20 \%)$, and $\mathrm{Au}+$ $\mathrm{Au} 200 \mathrm{GeV}(0-5 \%)$ collisions, with the corresponding PDG values. (c): The invariant mass of $\mathrm{K}^{+} \mathrm{K}^{-}$ in $\mathrm{Cu}+\mathrm{Cu}$ collisions at $\sqrt{s_{N N}}=200 \mathrm{GeV}$. (d): The $\phi$ meson mass as a function of $p_{T}$ for various centralities in $\mathrm{Cu}+\mathrm{Cu}$ collisions at $\sqrt{s_{N N}}=200 \mathrm{GeV}$.

Figures 3a and 3c show the invariant mass distributions of $\phi \rightarrow K^{+} K^{-}$in the non-single diffractive (NSD) $\mathrm{p}+\mathrm{p}, 0-5 \%$ central $\mathrm{Au}+\mathrm{Au}$, and $10-20 \%$ central $\mathrm{Cu}+\mathrm{Cu}$ collisions at $\sqrt{s_{N N}}=200 \mathrm{GeV}$ together with two MC simulation results using different momentum resolution settings. The lines show the non-relativistic Breit-Wigner functions superimposed on linear background functions. The measured $\phi$ meson masses are lower than the MC data at low $p_{T}$ in all collision systems and energies at $\sim 1 \sigma$ level in Figs. $3 \mathrm{~b}$ (upper panel) and 3d. The deviation from the PDG value is due to the multiple 
scattering energy loss of low $p_{T}$ tracks in the TPC. Since $p+p$ collisions also show the mass shifts from the MC data, this is unlikely the in-medium modification we are looking for. In Fig. $3 b$ (lower panel), the measured $\phi$ meson widths are presented and are larger than the MC simulation results [MC data set (1)]. If the momentum resolution for low $p_{T}$ kaons used in the simulations is increased by $50 \%$ [MC data set (2)], the $\phi$ meson width from the simulation reproduces the measured width from real data as shown by the open diamond points.

Figure 4 shows the invariant mass distributions of $\Lambda \pi^{ \pm}$(left panel) and $p K$ (right panel) after the mix-event background subtraction in $\mathrm{MB} \mathrm{p}+\mathrm{p}$ and central $\mathrm{Au}+\mathrm{Au}$ collisions at $\sqrt{s_{N N}}=200$ $\mathrm{GeV}$. In order to enhance the statistics the two charged $\Sigma^{* \pm}$ are combined for the $\mathrm{p}+\mathrm{p}$ collision data and all four charged $\Sigma^{* \pm}+\bar{\Sigma}^{* \pm}$ are combined for the $\mathrm{Au}+\mathrm{Au}$ collision data. For the $\Lambda^{*}$, particle and anti-particle, $\Lambda(1520)+\bar{\Lambda}(1520)$, are combined for the $\mathrm{p}+\mathrm{p}$ collision data and only $\Lambda(1520)$ is used for the $\mathrm{Au}+\mathrm{Au}$ collision data. Both $\Sigma^{*}$ and $\Lambda^{*}$ invariant mass distributions are fit with the non-relativistic Breit-Wigner function (red lines) plus linear background function (green lines). The fit results are summarized in Table 2. The measured masses and widths are in agreement with the PDG values within the errors except the masses of $\Sigma^{*}$, which shows small differences $\left(\sim 5 \mathrm{MeV} / \mathrm{c}^{2}\right)$.
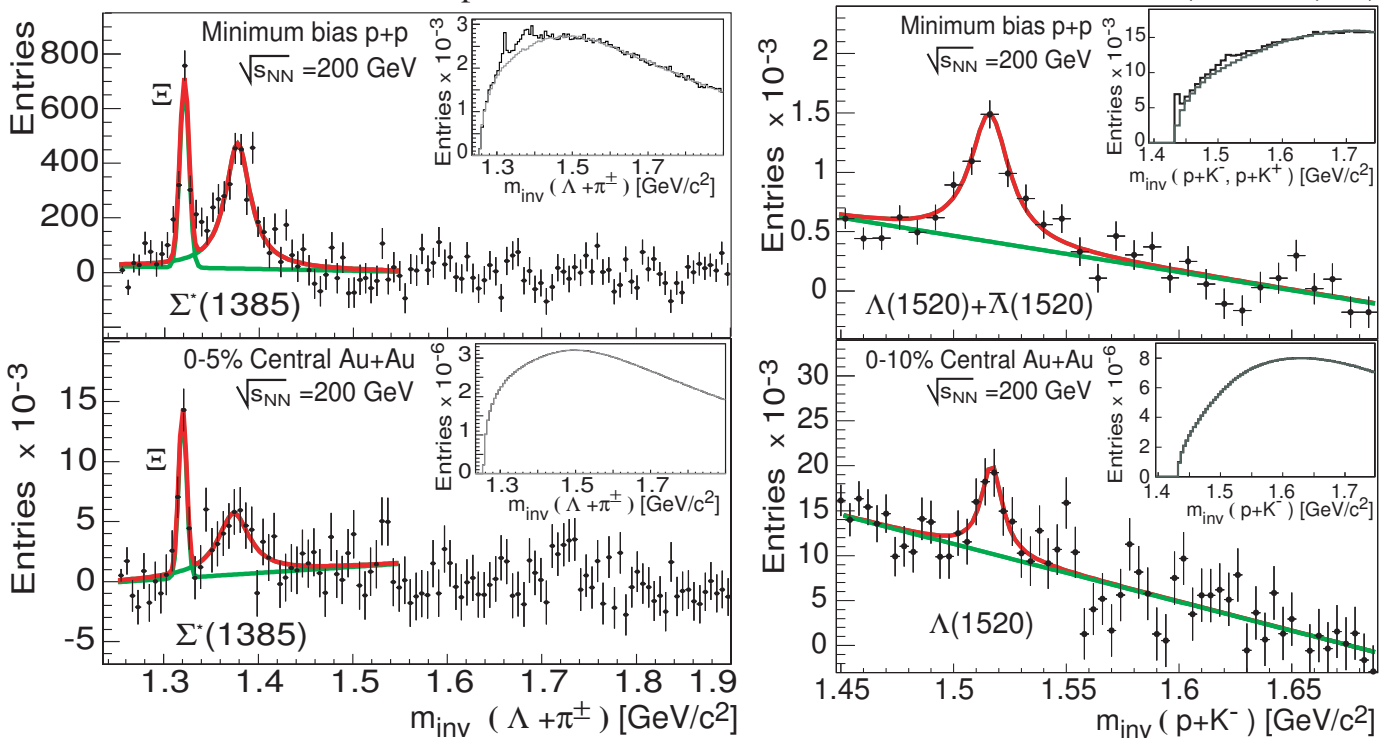

Fig. 4: Invariant mass distribution of $\Lambda \pi^{ \pm}$(left panel) and $p K$ (right panel) in MB p $+\mathrm{p}$ and central $\mathrm{Au}$ + Au collisions at $\sqrt{s_{N N}}=200 \mathrm{GeV}[12]$

Table 2: Measured $\Sigma^{*}$ and $\Lambda^{*}$ masses and widths with statistical and systematical uncertainties

\begin{tabular}{lll}
\hline particle & $\mathrm{M}\left(\mathrm{MeV} / \mathrm{c}^{2}\right)$ & $\Gamma\left(\mathrm{MeV} / \mathrm{c}^{2}\right)$ \\
\hline$\Sigma^{*}(\mathrm{p}+\mathrm{p})$ & $1376 \pm 3 \pm 3$ & $44 \pm 8 \pm 8$ \\
$\Sigma^{*}(\mathrm{Au}+\mathrm{Au})$ & $1375 \pm 5 \pm 3$ & $43 \pm 5 \pm 6$ \\
$\Lambda^{*}(\mathrm{p}+\mathrm{p})$ & $1516 \pm 2 \pm 2$ & $20 \pm 4 \pm 2$ \\
$\Lambda^{*}(\mathrm{Au}+\mathrm{Au})$ & $1516 \pm 2 \pm 2$ & $12 \pm 6 \pm 3$ \\
\hline
\end{tabular}

\subsection{Mean Transverse Momentum and dN/dy}

It is known that the $\left\langle p_{T}\right\rangle$ of the particles strongly depends on their masses as shown in Fig. 5. This can be explained by the hydrodynamic expansion picture [13] or the multiple minijets contributions [14]. From this dependency, resonance and non-resonance particles which have similar masses are expected to have similar $\left\langle p_{T}\right\rangle$. As showed in Fig. 6, however, the $\left\langle p_{T}\right\rangle$ of $K^{*}$ is higher than that of $\bar{p}$. A possible explanation is that the signal loss of resonances due to re-scattering of daughter particles in the hadronic medium occurs mostly at the low momentum region $\left(p_{T}<1 \mathrm{GeV} / \mathrm{c}\right)$ [15]. In consequence, 
the reconstructed transverse momentum spectra are expected to have higher inverse slope parameters and higher $\left\langle p_{T}\right\rangle$.

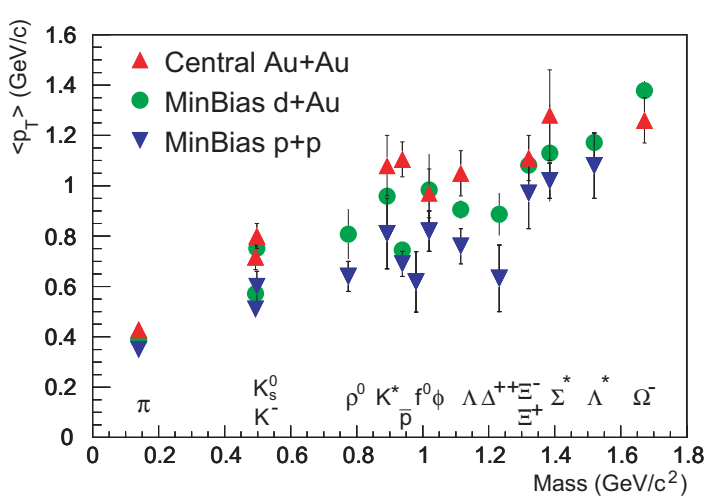

Fig. 5: $\left\langle p_{T}\right\rangle$ vs. particle masses at $\sqrt{s_{N N}}=200 \mathrm{GeV}$. The errors shown are the quadratic sum of statistical and systematical uncertainties. [16]

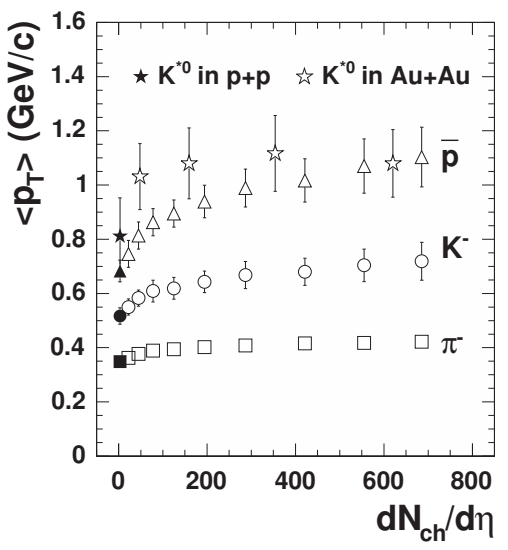

Fig. 6: $K^{* 0}\left\langle p_{T}\right\rangle$ as a function of $d N_{c h} / d \eta$ compared to that of stable particles in $\mathrm{MB}$ $\mathrm{p}+\mathrm{p}$ and $\mathrm{Au}+\mathrm{Au}$ collisions. [8]

Figure 7 shows the double ratios of resonance to stable particle for $\mathrm{Au}+\mathrm{Au}(\mathrm{Cu}+\mathrm{Cu})$ to $\mathrm{p}+\mathrm{p}$ collision systems $[5,9,11,12,18]$. The suppression of the ratios from unity in heavy ion collisions indicate extended hadronic medium in which the loss due to the re-scattering of the decay daughters is larger than the gain from the re-generation of the resonance particles. The suppression of the $K^{*}$ resonance in the hadronic decay channel $K^{*} \rightarrow K+\pi$ indicates that the re-scattering cross section, $\sigma_{\pi \pi}$, is larger than re-generation cross section, $\sigma_{\pi K}$. In contrast, the double ratio of the $\phi$ meson is consistent with unity owing to its relatively long lifetime and the negligible re-generation cross section, $\sigma_{K K}$. Since $\Lambda^{*}$ shows the largest suppression, it is most sensitive to this effect and more detailed study is desired. From these suppressions, the life time of the hadronic medium can be calculated [17].

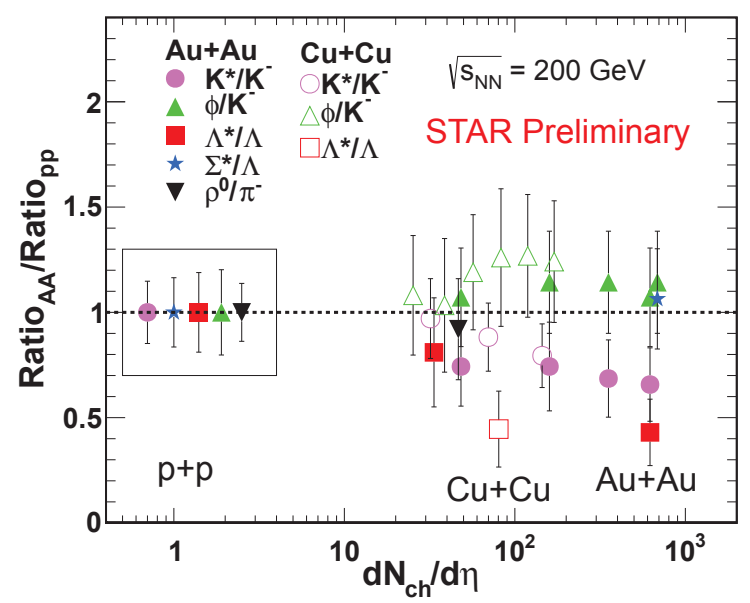

Fig. 7: Double ratios of resonance to stable particle for $\mathrm{Au}+\mathrm{Au}(\mathrm{Cu}+\mathrm{Cu})$ to $\mathrm{p}+\mathrm{p}$ collision systems at $\sqrt{s_{N N}}=200 \mathrm{GeV}$. The error bars show quadratic sum of statistical and systematic uncertainties. [5,9, $11,12,18]$ 


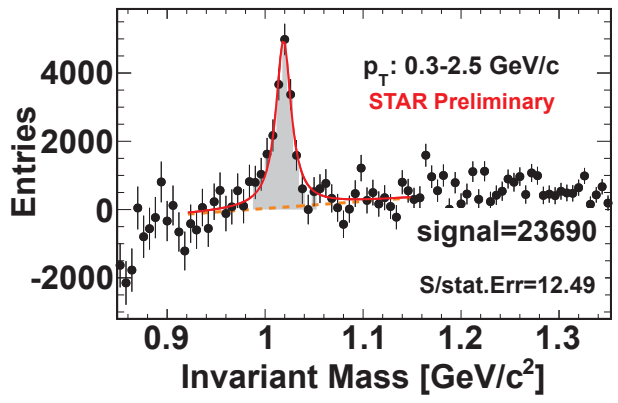

(a) $e^{+} e^{-}$invariant mass distribution

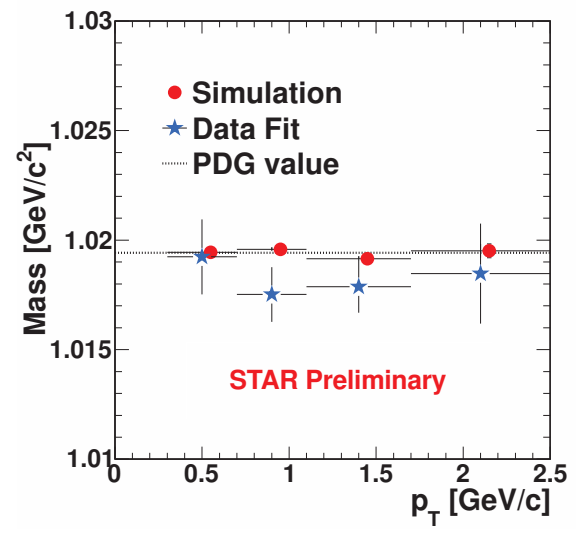

(c) $\phi$ meson mass vs. $p_{T}$

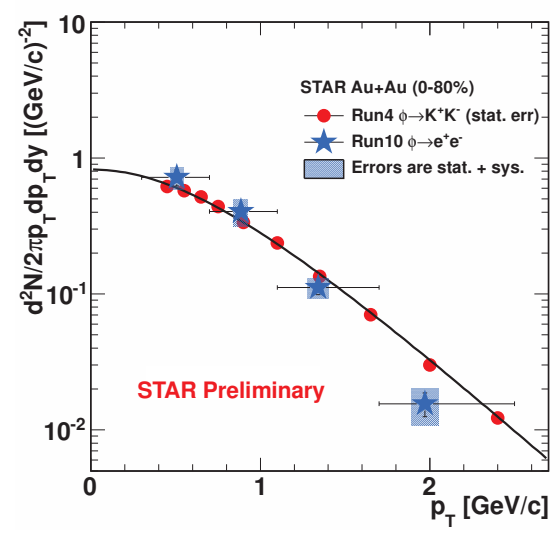

(b) Corrected $\phi$ meson yields comparison

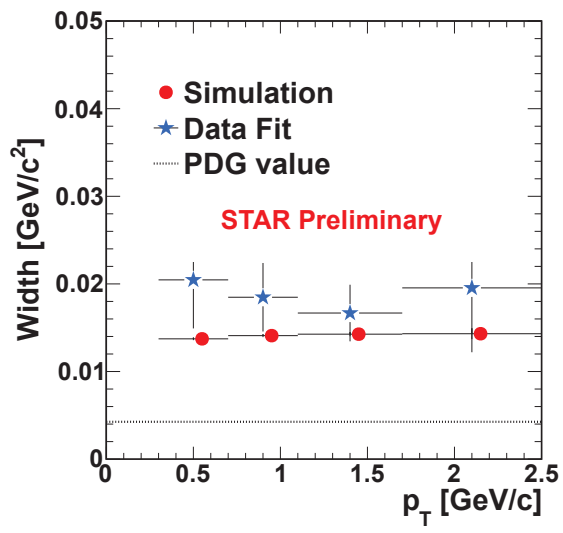

(d) $\phi$ meson width vs. $p_{T}$

Fig. 8: $\phi \rightarrow \mathbf{e}^{+} \mathbf{e}^{-}$in $\mathrm{Au}+$ Au collisions at $\sqrt{s_{N N}}=200 \mathrm{GeV}$

(a): The $p_{T}$ integrated $\phi \rightarrow e^{+} e^{-}$signals. The red solid line is the non-relativistic Breit-Wigner functions representing the $\phi$ signals and the orange dashed slope shows the residual backgrounds. (b): The corrected yields within rapidity $|y|<1$ for the $0-80 \%$ centrality events are represented by blue stars. The vertical bars are statistical errors and the boxes are the sum of systematic and statistical errors in quadrature. For comparison, the hadronic channel results [10] are plotted with red points and systematical error bars together with the exponential fit function (solid line) to the hadronic channel result. (c,d): mass (left) and width (right) vs. transverse momentum. The fit values and simulation results are respectively plotted in solid star and circles with statistical uncertainties. The dashed lines show the PDG values. [19]

\section{Resonances from leptonic decay channels}

Figure 8a shows the invariant mass distribution of the $e^{+} e^{-}$pairs after the mixed-events background subtraction in the transverse momentum range of 0.3 to $2.5 \mathrm{GeV} / \mathrm{c}$ for $0-80 \%$ centrality events from $\mathrm{Au}+\mathrm{Au}$ collisions at $\sqrt{s_{N N}}=200 \mathrm{GeV}$. Figure $8 \mathrm{~b}$ shows the $\phi \rightarrow e^{+} e^{-}$invariant yields shown by star symbols while solid circles represent STAR published $\phi \rightarrow K^{+} K^{-}$results [10]. The $\phi$ measurements in di-electron decay channel are consistent with the STAR published results within $1.2 \sigma$, if we take into account the $10 \%$ systematical uncertainty of the published result. The fit results of the mass and decay width of the $\phi(1020)$ meson in each $p_{T}$ bin are presented with blue points in Figs. $8 \mathrm{c}$ and $8 \mathrm{~d}$, 
respectively. The error bars are statistical errors (fit parameter errors) only. Simulation results and PDG values are plotted with red points and dashed lines, respectively. The fit, simulation, and PDG values of the mass are consistent within $\sim 1.5 \sigma$. On the other hand, the fit decay widths show significant discrepancy from the PDG value. This width broadening, however, can be explained by the momentum resolution of the detector entirely because the simulation results also shows the broadening.

The $\omega \rightarrow e^{+} e^{-}$is also measured in STAR [20,21]. The invariant mass distributions of the $e^{+} e^{-}$ pairs after the mixed-events background subtraction in $\mathrm{p}+\mathrm{p}$ and $\mathrm{Au}+\mathrm{Au}$ collisions at $\sqrt{S_{N N}}=$ $200 \mathrm{GeV}$ are presented in Fig. 9. The signal and residual background line shapes are obtained from cocktail simulations and they are fit to the data to obtain signal yields [21]. Figure 9 (right panel) shows corrected transverse momentum spectra of $\omega \rightarrow e^{+} e^{-}$in $\mathrm{p}+\mathrm{p}$ and $\mathrm{Au}+\mathrm{Au}$ collisions at $\sqrt{s_{N N}}=200$ compared to the published PHENIX result [22] and predictions from Tsallis Blast-Wave function fits [23]. The results agree with the previous result in $\mathrm{p}+\mathrm{p}$ collisions and the predictions in both $\mathrm{p}+\mathrm{p}$ and $\mathrm{Au}+\mathrm{Au}$ collisions.

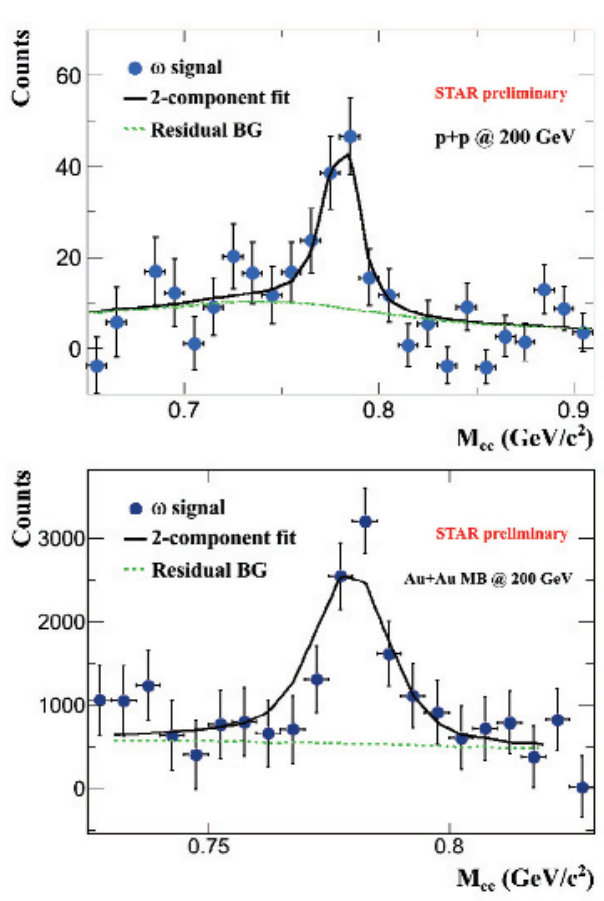

(a) $e^{+} e^{-}$invariant mass distribution

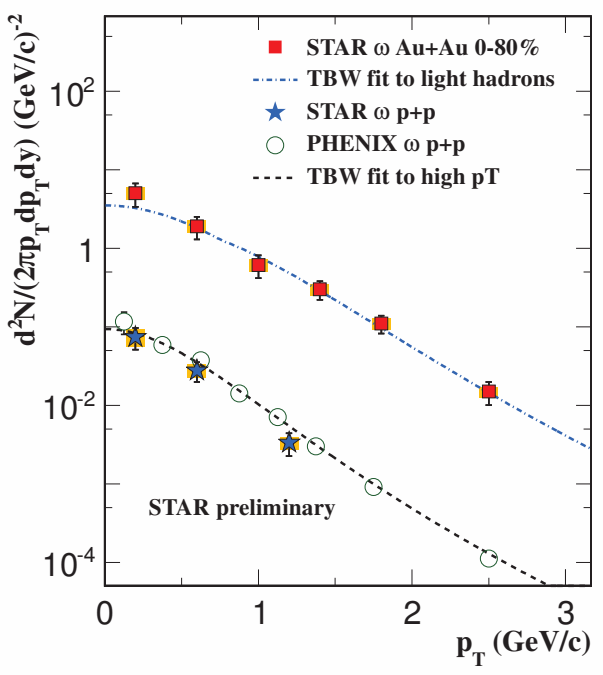

(b) Corrected $\omega$ yields [21]

Fig. 9: $\omega \rightarrow \mathbf{e}^{+} \mathbf{e}^{-}$in $\mathbf{p}+\mathbf{p}$ and $\mathbf{A u}+\mathbf{A u}$ collisions at $\sqrt{s_{N N}}=200 \mathbf{G e V}$

(a): The $p_{T}$ integrated invariant mass signals in $\mathrm{p}+\mathrm{p}$ (upper panel) and $\mathrm{Au}+\mathrm{Au}$ (lower panel) collisions. The black solid lines and green dashed lines in both figures respectively represent $\omega$ signals and residual backgrounds obtained from cocktail simulation [21]. (b): The $\omega \rightarrow e^{+} e^{-}$invariant yield, divided by its B.R., in NSD p $+\mathrm{p}$ and $\mathrm{Au}+\mathrm{Au}$ collisions at $\sqrt{s_{N N}}=200 \mathrm{GeV}$ [20]. The circles represent PHENIX published results [22]. The bars and boxes are statistical and systematic uncertainties, respectively. The black dashed line represents the yields of $\omega$ from the Tsallis Blast-Wave function fit.

\section{Summary}

In summary, resonance particles are a useful probe to study the hadronic medium in heavy ion collisions. Many resonance particles are measured in several collision systems $(p+p, d+\mathrm{Au}, \mathrm{Cu}+\mathrm{Cu}$, and 
$\mathrm{Au}+\mathrm{Au}$ ) and energies (only $\sqrt{s_{N N}}=62.4$ and $200 \mathrm{GeV}$ are presented) in STAR. The $\left\langle p_{T}\right\rangle$ of $K^{*}$ shows the increase compared to that of $\bar{p}$, although expected to have similar $\left\langle p_{T}\right\rangle$. This could be explained by the re-scattering contribution from hadronic medium. The double ratio of $K^{*} / K$ and $\Lambda^{*} / \Lambda$ yields show suppression with centrality while $\phi / K^{-}$stays at unity. This comes from differences in their lifetimes and cross sections of their daughter particles. New measurements of $\phi$ and $\omega$ from leptonic decay channels are presented. Both results from leptonic decay channels and from hadronic decay channels are consistent. No signature of the chiral symmetry restoration is observed.

\section{References}

1. G. E. Brown and M. Rho, Phys. Rev. Let. 66, 2720 (1991)

2. R. Rapp, J. Wambach, and H. van Hees, arXiv:0901.3289v1 [hep-ph]

3. B. Bonner et al., Nucl. Instr. Meth. A 508, 181 (2003); M. Shao et al., Nucl. Instr. Meth. A 492, 344 (2002); J. Wu et al., Nucl. Instr. Meth. A 538, 243 (2005)

4. J. Beringer et al. (Particle Data Group), Phys. Rev. D86, 010001 (2012)

5. J. Adams et al., Phys. Rev. Lett. 92, 092301 (2004)

6. P. Fachini (STAR collaboration), Nucl.Phys. A830, 837c (2009)

7. R. Rapp, Nucl.Phys. A 725, 254 (2003)

8. J. Adams et al., Phys. Rev. C71, 064902 (2005)

9. M. M. Aggarwal et al., Phys. Rev. C84, 034909 (2011)

10. B. I. Abelev et al., Phys. Rev. C79, 064903 (2009)

11. B. I. Abelev et al., Phys. Lett. B 673, 183 (2009)

12. B. I. Abelev et al., Phys. Rev. Lett. 97, 132301 (2006)

13. P. F. Kolb and R. Rapp, Phys. Rev. C 67, 044903 (2003)

14. A.Dumitru and C. Spieles, Phys. Lett. B 446, 326 (1999)

15. M. Bleicher, J. Aichelin, Phys. Lett. B 530, 81 (2002)

16. B. I. Abelev et al., Phys. Rev. C78, 044906(2008)

17. C. Markert, G. Torrieri, and J. Rafelski, arXiv:hep-ph/0206260v2

18. Masayuki Wada (STAR collaboration), APS April Meeting 2011

19. Masayuki Wada (STAR collaboration), A. Phys. Pol. B Supp. 5249 (2012)

20. Bingchu Huang (STAR collaboration), A. Phys. Pol. B Supp. 5471 (2012)

21. L. Adamczyk et al., Phys. Rev. C 86, 024906 (2012)

22. A. Adare et al., Phys. Rev. D 83, 052004 (2011)

23. Z.Tang et al., arXiv:1101.1912v3 [nucl-ex] (2011) 